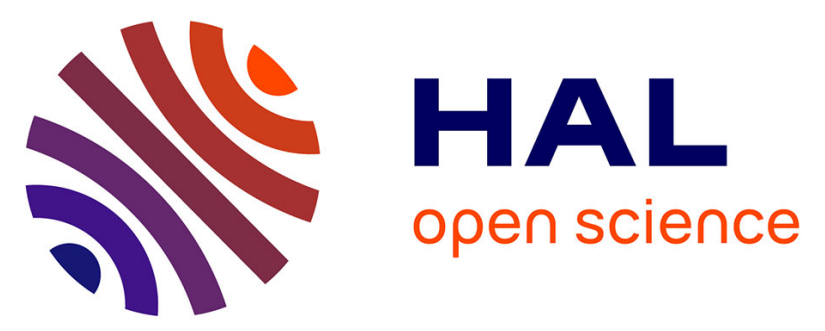

\title{
A relationship between non-exponential stress relaxation and delayed elasticity in the viscoelastic process in amorphous solids: Illustration on a chalcogenide glass \\ Yann Gueguen, Vincent Keryvin, Tanguy Rouxel, Mickaël Le Fur, Hervé \\ Orain, Bruno Bureau, Catherine Boussard-Plédel, Jean-Christophe \\ Sangleboeuf
}

\section{To cite this version:}

Yann Gueguen, Vincent Keryvin, Tanguy Rouxel, Mickaël Le Fur, Hervé Orain, et al.. A relationship between non-exponential stress relaxation and delayed elasticity in the viscoelastic process in amorphous solids: Illustration on a chalcogenide glass. Mechanics of Materials, 2015, 85, pp.47-56. 10.1016/j.mechmat.2015.02.013 . hal-01153240

HAL Id: hal-01153240

https://hal-univ-rennes1.archives-ouvertes.fr/hal-01153240

Submitted on 19 May 2015

HAL is a multi-disciplinary open access archive for the deposit and dissemination of scientific research documents, whether they are published or not. The documents may come from teaching and research institutions in France or abroad, or from public or private research centers.
L'archive ouverte pluridisciplinaire HAL, est destinée au dépôt et à la diffusion de documents scientifiques de niveau recherche, publiés ou non, émanant des établissements d'enseignement et de recherche français ou étrangers, des laboratoires publics ou privés. 


\title{
A relationship between non-exponential stress relaxation and delayed elasticity in the viscoelastic process in amorphous solids: illustration on a chalcogenide glass
}

\author{
Yann Gueguen ${ }^{\mathrm{a}}$, Vincent Keryvin ${ }^{\mathrm{b}, \mathrm{a}}$, Tanguy Rouxel ${ }^{\mathrm{a}}$, Mickaël Le Fur ${ }^{\mathrm{a}}$, Hervé Orain ${ }^{\mathrm{a}}$, Bruno Bureau ${ }^{\mathrm{c}}$, Catherine \\ Boussard-Plédel ${ }^{\mathrm{c}}$, Jean-Christophe Sangleboeuf ${ }^{\mathrm{c}}$ \\ ${ }^{a}$ IPR UMR UR1-CNRS 6251, Department Mechanics and Glasses, Université de Rennes 1, Campus de Beaulieu, 35042 Rennes Cedex, France \\ ${ }^{b}$ LIMATB EA 4250, Université de Bretagne Sud, Rue de Saint Maudé, 56321 Lorient Cedex, France \\ ${ }^{c}$ Equipe Verres et Céramiques, UMR-CNRS 6226 Sciences Chimiques de Rennes, Université de Rennes 1, Campus de Beaulieu, 35042 Rennes Cedex, France
}

\begin{abstract}
Inorganic glasses are viscoelastic materials since they exhibit, below as well as above their glass transition temperature, a viscoelastic deformation under stress, which can be decomposed into a sum of an elastic part, an inelastic (or viscous) part and a delayed elastic part. The delayed elastic part is responsible for the non-linear primary creep stage observed during creep tests. During a stress relaxation test, the strain, imposed, is initially fully elastic, but is transformed, as the stress relaxes, into an inelastic and a delayed elastic strains. For linear viscoelastic materials, if the stress relaxation function can be fitted by a stretched exponential function, the evolution of each part of the strain can be predicted using the Boltzmann superposition principle. We develop here the equations of these evolutions, and we illustrate their accuracy by comparing them with experimental evolutions measured on $\mathrm{GeSe}_{9}$ glass fibers. We illustrate also, by simple equations, the relationship between any kind of relaxation function based on additive contribution of different relaxation processes and the delayed elastic contribution to stress relaxation: the delayed elasticity is directly correlated to the dispersion of relaxations times of the processes involved during relaxation.
\end{abstract}

Keywords:

delayed elasticity, anelasticity, stretch exponent, linear viscoelasticity, chalcogenide glasses

\section{Introduction}

Many models (please see (Phillips, 1996) and references ${ }^{29}$ therein) have been proposed to explain the non-exponential ${ }^{30}$ relaxation in amorphous solids, and to give an "unified" theory, ${ }^{31}$ valid for any kind of relaxation, including stress relaxation. ${ }^{32}$ Nevertheless, the "delayed elasticity", that inevitably emerges ${ }^{33}$ when the stress relaxation is not exponential, is not often dis- ${ }^{34}$ cussed (see (Goldstein, 1969) where this problem is detailed) ${ }^{35}$ despite its preponderance during the first stages of relaxation. ${ }^{36}$ It is known, since more than a century and a half (Weber, ${ }^{37}$ 1835), that the primary or transient creep stage of almost all ${ }^{38}$ kind of materials is due to delayed elasticity. It is not only ${ }^{39}$ due to a possible non-equilibrium viscosity, as astonishingly ${ }^{40}$ suggested by the ASTM standard for viscosity measurements ${ }^{41}$ (ASTM C1350-96, 1996), since glasses under equilibrium also ${ }^{42}$ exhibit primary creep stage. Delayed elasticity is also named ${ }^{43}$ "anelasticity" (mainly for metals (Zener, 1948)), or "retarded ${ }^{44}$ elasticity" (Goldstein, 1969) and was originally named "elastic ${ }^{45}$ aftereffect" ("elastiche nachwirkung" (Boltzmann, 1876)). ${ }^{46}$ The delayed elastic deformation is a reversible deformation ${ }^{47}$ ("elastic") which does not recover instantaneously ("delayed" 48 or "retarded") when the stress is released. The history of the 49 investigation of the "aftereffect", taking its origins in Göttingen 50 with Weber and Kohlrausch, has been nicely summarized by 51 Bendler (1984). The primary creep stage is supposed to occur 52 in any kind of material, including ceramics, crystalline metallic ${ }_{53}$ materials, polymers, inorganic glasses, metallic glasses, as well as biomaterials. Thus, any kind of material is supposed to exhibit, during creep flow, delayed elasticity. Since nowadays a lot of commercial machines are available for measuring the steady state viscosity from the creep rate, it is sometimes forgotten that the creep rate is not solely controlled by the viscosity. During the primary creep stage, the creep rate is mainly controlled by the delayed elasticity, and the viscosity parameter can be measured only when the stationary creep stage is reached. This, for inorganic glasses, below their glass transition temperature, can take hours to days. Consequently, a "continuous viscosity measurement", in the glass transition range, below or just above (down to a viscosity of, let say, $10^{9-10} \mathrm{~Pa} . \mathrm{s}$ ), based on the continuous creep rate measurement, using continuous heating or cooling (no matter how slow it is), does not even make sense, and will obviously tend to give an "anomalous" apparent viscosity. In other words, non-isothermal viscosity measurements are necessarily wrong, because of the delayed elasticity. It underlines the necessity of a better knowledge of this delayed elastic deformation.

If it is quite straightforward to measure the delayed elasticity during a creep-recovery test, it becomes more delicate to evaluate it during a relaxation test. Wiechert (1893) is probably the first who has suggested that the delayed elasticity takes its origin in the non-exponentiality of the stress relaxation. A non-exponential relaxation can be modelled by a gen- 
eralized Maxwell model (Maxwell, 1868) (or more precisely ${ }_{102}$ a "Maxwell-Wiechert model" (Wiechert, 1893)), where the re-103 laxation modulus is decomposed into a Prony series. The equa-104 tions, depicting the correlation between a non-exponential re-105 laxation corresponding to a Maxwell-Wiechert model and the ${ }_{106}$ primary creep stage (including the delayed elasticity), have ${ }_{107}$ been set by Bennewitz and Rötger (see (Simha, 1942)). The ${ }_{108}$ Maxwell-Wiechert model is efficient to describe the viscoelastic behavior of silica glasses (Duffrène, Gy, Burlet, and Piques, 1997), but it requires a large number of parameters as com- ${ }^{109}$ pared to the well-known stretched exponential or "KWW" (Kohlrausch-Williams-Watts) function (Kohlrausch, 1854):

$$
\varphi(t)=\frac{Q(t)-Q_{\infty}}{Q(0)-Q_{\infty}}=\exp \left(-\left(\frac{t}{\tau_{0}}\right)^{\beta}\right)
$$

where $\varphi$ is the relaxation function, describing the normalized ${ }^{115}$ relaxation of a quantity $Q$ (here the shear stress), $Q_{\infty}$ being its asymptotic value at $t \rightarrow+\infty$. $\beta$ is the stretch exponent, and $\tau_{0}{ }^{116}$ is a characteristic time, related to the average (or "Maxwell's") relaxation time $\tau_{a}$ by the following relation:

$$
\tau_{a}=\int_{0}^{+\infty} \varphi(t) d t=\frac{\Gamma(1 / \beta)}{\beta} \tau_{0}
$$

where $\Gamma$ is the Gamma function: $\Gamma(x, y)=\int_{y}^{\infty} s^{x-1} e^{-s} d s_{121}$ and $\Gamma(x)=\Gamma(x, 0)$. The average relaxation time corresponds to the average lifetime of an atom/molecule or group of ${ }_{122}$ atoms/molecules in their equilibrium position (here when they ${ }_{123}$ are shifted by the stress) (Frenkel, 1926). The stretched exponential function (SEF) has various advantages. The main one ${ }_{124}$ is that it has only two parameters to describe the full relaxation spectrum. The second one is that the "departure" from the exponentiality is directly signed by the stretch exponent $\beta$. So, ${ }_{126}$ according to the idea of Wiechert, the delayed elasticity is directly signed by $\beta$. The SEF has been widely used to describe ${ }_{127}$ various kind of relaxation processes (see for example (Phillips, 1996)), but this function is not physically sound, first because ${ }^{128}$ regarding its time derivation:

$$
\frac{d \varphi(t)}{d t}=-\varphi(t)\left(\frac{t}{\tau_{0}}\right)^{\beta} \beta t^{-1}
$$

the relaxation rate tends to $-\infty$ when $t \rightarrow 0^{+}$, for $0<\beta<1{ }_{{ }_{133}}{ }_{132}$ Duffrène et al. (1997) have also pointed out the inadequacy ${ }_{134}$ of the SEF to describe the viscoelastic behavior of soda-lime- ${ }_{135}$ silica glasses. Additionally, they have shown that if we assume ${ }_{136}$ that the shear relaxation function corresponds to a SEF, the ${ }_{137}$ primary creep stage can not be fitted by another SEF, as it is ${ }_{138}$ often done.

In spite of the weaknesses of the SEF, we will show here, experimentally, how much the idea of Wiechert is correct: the delayed elasticity is only connected to the stretch exponent. The SEF is largely used in the literature mainly in order to describe the relaxation kinetic, but what is not often considered is its ability to describe the detailed mechanisms of relaxation, such ${ }^{142}$ as, for stress relaxation, the delayed elasticity and the inelasticity. We will illustrate, through relaxation-recovery tests on a viscoelastic material, how much efficient is the SEF to predict the evolution of the delayed elasticity. Then, we will show how we can interpret the relationship between any kind of relaxation function decomposable into a sum of exponential function (such as the SEF) and the delayed elasticity.

\section{Theory}

\subsection{Linear viscoelasticity}

Let us consider a linear viscoelastic body, undergoing a shear distortion, evolving over time: $\gamma(t)$, in the framework of the small strain assumption. According to the Boltzmann superposition principle (Boltzmann, 1876), the resulting shear stress is $\sigma$ :

$$
\sigma(t)=\int_{-\infty}^{t} G(t-s) \frac{d \gamma(s)}{d s} d s
$$

$G$ is the shear relaxation modulus of the viscoelastic body. Of course, considering the distortion as something imposed is just a point of view, and consequently, if we consider instead the stress as imposed, the resulting shear distortion is:

$$
\gamma(t)=\int_{-\infty}^{t} J(t-s) \frac{d \sigma(s)}{d s} d s
$$

$J$ is the shear creep compliance. $J$ and $G$ are correlated by a Duhamel convolution equation (Ferry, 1980):

$$
t=\int_{0}^{t} G(t-s) \times J(s) d s=\int_{0}^{t} G(s) \times J(t-s) d s
$$

Or, considering the well-known Lee (1955)-Mandel correspondence principle, the equations, for linear elasticity being:

$$
\gamma=\mu^{-1} \sigma \text { and } \sigma=\mu \gamma \text { so that: } \mu^{-1} \mu=1
$$

where $\mu$ is the elastic shear modulus, we obtain for linear viscoelasticity, considering $G$ and $J$ as the viscoelastic counterparts of $\mu$ and $\mu^{-1}$ respectively:

$$
\gamma^{*}=J^{*} \sigma^{*} \text { and } \sigma^{*}=G^{*} \gamma^{*} \text { so that: } J^{*} G^{*}=1
$$

where $f^{*}$ is the Laplace-Carson transform of $f: f^{*}=s \times \mathcal{L}(f)$, $\mathcal{L}(f)$ being the Laplace transform of $f$ and $s$ the variable in the Laplace $s$-domain.

The shear creep compliance $J$ is often explicitly divided into a sum of three components: elastic $J_{e}$ time-independent, delayed elastic $J_{d}(t)$ which converges to a time-independent value when $t \rightarrow+\infty$ and inelastic $J_{\eta}$ a linear function of time:

$$
J_{e}=\frac{1}{\mu} \text { and } J_{\eta}(t)=\frac{t}{\eta}
$$

where $\eta$ is the shear viscosity. Using Eq.(6), we also obtain:

$$
J_{d}(t)=\mathcal{L}^{-1}\left(\frac{1}{s^{2} \mathcal{L}(G(t))}\right)-\frac{t}{\eta}-\frac{1}{\mu}
$$


This decomposition of $J$ is clearly assumed for various well-183 known models. This is the case for the Burger's model, the ${ }_{184}$ generalized Burger's model, and for any kind of model or func-185 tion describing $J$ with a retardation function (a function cor-186 responding to $\left.\phi(t)=1-J_{d}(t) / J_{d}(0)\right)$. Other models, describing the shear relaxation modulus, such as the Maxwell-187 Wiechert model, implicitly assume this division of $J$, since $_{188}$ the Burger's model is equivalent to a Maxwell-Wiechert model ${ }_{189}$ with two cells. Accordingly, the shear distortion, itself, can be divided into three components, associated with $J_{e}, J_{d}$ and $J_{\eta}$ : an elastic distortion $\gamma_{e}$, reversible, a delayed elastic distortion $\gamma_{d}$, reversible but with delay, and an inelastic distortion $\gamma_{\eta}$, irreversible. We can write, using the Boltzmann superposi- ${ }^{19}$ tion principle (Boltzmann, 1876) or the Lee-Mandel correspon- ${ }^{192}$ dence principle:

$$
\gamma_{c}(t)=\int_{-\infty}^{t} J_{c}(t-s) \frac{d \sigma(s)}{d s} d s \text { or } \gamma_{c}^{*}(s)=J_{c}^{*}(s) \sigma^{*}(s)
$$

where $c=e, d$ or $\eta$. With:

$$
\gamma(t)=\gamma_{e}(t)+\gamma_{d}(t)+\gamma_{\eta}(t) \& J(t)=J_{e}(t)+J_{d}(t)+J_{\eta}(t)(12)_{197}
$$

So, we can also write:

$$
\begin{aligned}
& \gamma_{e}(t)=\frac{\sigma(t)}{\mu} \\
& \gamma_{\eta}(t)=\int_{-\infty}^{t} \frac{\sigma(s)}{\eta} d s \\
& \gamma_{d}(t)=\gamma(t)-\gamma_{e}(t)-\gamma_{\eta}(t)
\end{aligned}
$$

Regarding these equations, and assuming $\gamma_{\eta}(t)=0$ if $t<0$, we can deduce that:

$$
\eta=\frac{\sigma(t)}{\dot{\gamma}_{\eta}(t)}
$$

This is the conventional "newtonian viscosity". But, using Eq.(6) we also have the following relation, known as the "Ferry's relation" (Ferry, 1980):

$$
\eta=\int_{0}^{+\infty} G(t) d t
$$

\subsection{Delayed elasticity}

Using Eq.(10) and (11), we can see that there is no delayed ${ }^{213}$ elasticity, for any kind of stress history, only if $J_{d}(t)=0$. It leads to (Eq.(6) \& (9)):

$$
J(t)=\frac{1}{\mu}+\frac{t}{\eta} \text { and } G(t)=\mu \exp \left(-\frac{t \mu}{\eta}\right)
$$

The average shear relaxation time $\tau_{a}$, according to the Maxwell's relation (Maxwell, 1868) corresponds to: $\tau_{a}=\eta / \mu_{\cdot_{217}}$ In other words, the only possibility to observe no delayed elasticity is to have an exponential relaxation. It clearly illustrates the assertion of Wiechert (1893) that the delayed elas- ${ }_{219}$ ticity comes from a non-exponential relaxation. The equation ${ }_{220}$ of Simha (1942), giving the relationship between a creep curve and the distribution of relaxation times leads to the same conclusion. As a matter of fact, if the shear relaxation modulus ${ }^{221}$ of a linear viscoelastic body is described by a SEF, for any $0<\beta<1$, when this body deforms non-elastically (whatever if the stress is constant or not), a part of the deformation is or has been delayed elastic.

\subsection{Stretched exponential relaxation}

Let us now assume that the shear relaxation modulus can be fitted by a SEF.

$$
G(t)=\mu \varphi(t) \text { where } \varphi(t)=\exp \left(-\left(\frac{t}{\tau_{0}}\right)^{\beta}\right)
$$

where $0<\beta<1$. Using a SEF, it is not possible to obtain an analytic form of $J_{d}$ in Eq.(10), since the SEF has no Laplacetransform (Duffrène et al., 1997). But, we can assume:

$$
J_{d}(t)=\frac{1}{\mu_{d}}(1-\phi(t))
$$

where $\phi$ is a retardation function $\left(\phi(0)=1\right.$ and $\left.\lim _{t \rightarrow+\infty} \phi(t)=0\right)$, and $\mu_{d}^{-1}$ corresponds to the asymptotic value of $J_{d}(t)$ (when $t \rightarrow$ $+\infty)$. According to Duffrène et al. (1997):

$$
\frac{1}{\mu_{d}}=\frac{1}{\mu}\left(\frac{\Gamma(1+2 / \beta)}{(\Gamma(1+1 / \beta))^{2}}-1\right)
$$

Thus, if we perform a creep test (constant stress $\sigma_{0}$ ), the delayed elastic distortion continuously increases over time, and depends on $\sigma_{0}, \mu, \beta$. According to (Eq.(11)):

$$
\gamma_{d \infty}=\lim _{t \rightarrow+\infty} \gamma_{d}(t)=\frac{\sigma_{0}}{\mu_{d}}=\gamma_{0}\left(\frac{\Gamma(1+2 / \beta)}{(\Gamma(1+1 / \beta))^{2}}-1\right)
$$

where $\gamma_{0}=\sigma_{0} / \mu$, is the initial shear distortion, fully elastic. Considering a constant $\gamma_{0}$, this equation illustrates that $\gamma_{d \infty}$ is maximum if $\beta \rightarrow 0$ and minimum if $\beta \rightarrow 1$. This is illustrated on Figure 1. The equation of Duffrène et al. (1997) illustrates that the stretch exponent is an indicator of the delayed elasticity level.

Now, if instead of performing a creep test, we perform a shear relaxation test on a linear viscoelastic body, so that:

$$
\gamma(t)=\gamma_{0} \mathcal{H}(t)
$$

$\mathcal{H}$ being the Heaviside function. Assuming that the relaxation function is a SEF, we have, using Eq.(4) and (17), for $t \geq 0$ :

$$
\gamma_{e}(t)=\gamma_{0} \varphi(t)=\gamma_{0} \exp \left(-\left(\frac{t}{\tau_{0}}\right)^{\beta}\right)
$$

And according to the Hooke's law: $\sigma(t)=\mu \gamma_{e}(t)$. Using Eq.(13), we have:

$$
\gamma_{\eta}(t)=\frac{\int_{0}^{t} \sigma(s) d s}{\eta}=\frac{\mu \gamma_{0} \int_{0}^{t} \varphi(s) d s}{\eta}
$$

Using the symbolic computation Mathematica software (from Wolfram Research, Inc., Champaign, Illinois, version 5.2 edition, 2005),

$$
\int_{0}^{t} \varphi(s) d s=\frac{\tau}{\beta}\left(\Gamma\left(\frac{1}{\beta}\right)-\Gamma\left(\frac{1}{\beta},\left(\frac{t}{\tau_{0}}\right)^{\beta}\right)\right)
$$




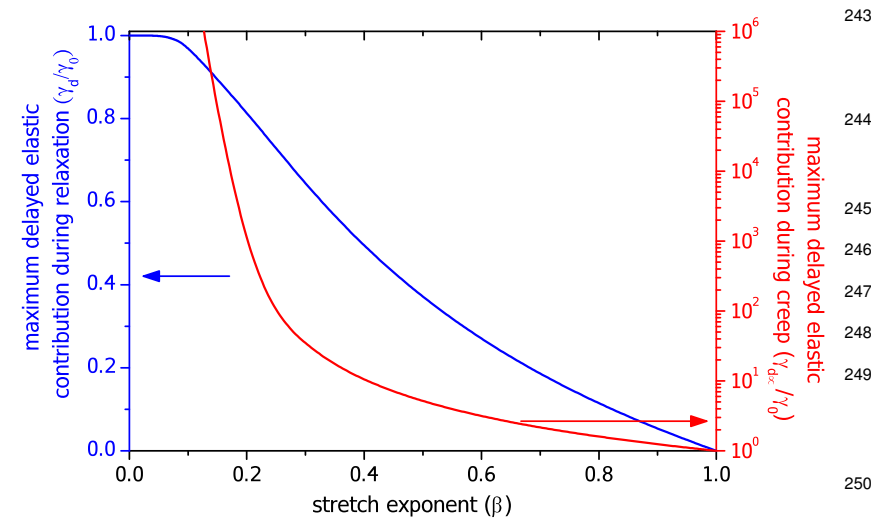

251

Figure 1: Evolution of the maximum relative (normalized by the initial dis- ${ }^{252}$ tortion $\gamma_{0}$ ) delayed elastic distortion during conventional mechanical tests for $^{253}$ viscoelastic materials, as a function of the stretch exponent $(\beta)$ of stress relax-254 ation. On the left axis, during a relaxation test $\left(\gamma_{d} / \gamma_{0}\right.$, blue) and on the right ${ }_{255}$ axis during a creep test $\left(\gamma_{d \infty} / \gamma_{0}\right.$, red).
Since $G(t)=\mu \varphi(t)$, using Eq.(2) \& (15), we have $\eta=258$ $\mu \tau_{a}$ (well known "Maxwell's relationship"). Substituting, in259 Eq.(23), $\eta$ by this latter expression and $\int_{0}^{t} \varphi(s) d s$ by the expres- ${ }^{260}$ sion found in Eq.(24), we have:

$$
\gamma_{\eta}(t)=\gamma_{0}\left(1-\frac{\Gamma\left(1 / \beta,\left(\frac{t}{\tau_{0}}\right)^{\beta}\right)}{\Gamma(1 / \beta)}\right)
$$

Finally, using Eq.(13):

$$
\gamma_{d}(t)=\gamma_{0}\left(\frac{\Gamma\left(1 / \beta,\left(\frac{t}{\tau_{0}}\right)^{\beta}\right)}{\Gamma(1 / \beta)}-\varphi(t)\right)
$$

In order to eliminate $\tau_{0}$ in these latter equations, to highlight ${ }^{272}$ the role of the exponent $\beta$, let us introduce:

$$
\Lambda=\frac{\sigma(0)-\sigma(t)}{\sigma(0)}=1-\varphi(t)
$$

$\Lambda$ is the relative fraction of stress relaxed. The total shear dis- ${ }^{277}$ tortion is $\gamma=\gamma_{0}$ for $t \geq 0$, so that the relative fraction of each ${ }^{278}$ distortion component is:

$$
\begin{aligned}
& \frac{\gamma_{e}(\Lambda)}{\gamma_{0}}=1-\Lambda \\
& \frac{\gamma_{\eta}(\Lambda)}{\gamma_{0}}=1-\frac{\Gamma(1 / \beta,-\ln (1-\Lambda))}{\Gamma(1 / \beta)} \\
& \frac{\gamma_{d}(\Lambda)}{\gamma_{0}}=\Lambda-1+\frac{\Gamma(1 / \beta,-\ln (1-\Lambda))}{\Gamma(1 / \beta)}
\end{aligned}
$$

We can calculate when the delayed elasticity will reach its max-287 imum, when $\varphi$ is a SEF, by solving $d \gamma_{d} / d \Lambda=0$. The maximum $_{288}$ is obtained, if $0<\beta<1$ when:

$$
\Lambda_{M}=1-\exp \left(-(\Gamma(1 / \beta))^{\frac{\beta}{1-\beta}}\right)
$$

Inserting Eq.(31) in Eq.(30), we obtain the maximum delayed elastic distortion during shear relaxation $\left(\gamma_{d}\left(\Lambda_{M}\right) / \gamma_{0}\right)$ :

$$
\frac{\gamma_{d}\left(\Lambda_{M}\right)}{\gamma_{0}}=\frac{\Gamma\left(1 / \beta, \Gamma(1 / \beta)^{\frac{\beta}{1-\beta}}\right)}{\Gamma(1 / \beta)}-\exp \left(-\Gamma(1 / \beta)^{\frac{\beta}{1-\beta}}\right)
$$

This maximum is only a function of $\beta$ : neither $\tau_{0}$ nor $\mu$ appears in this equation. The stretch exponent is the only indicator of the maximum delayed elastic contribution to shear stress relaxation. Therefore, as for creep, we will see that the delayed elasticity is maximum if $\beta \rightarrow 0$ and minimum (null) if $\beta \rightarrow 1$.

\section{Experiments}

In order to challenge all the equations we have developed in the previous section, we have performed shear relaxation and strain recovery tests on $\mathrm{GeSe}_{9}$ glass fibers by a torsional method. The glass and fibers synthesis is described in (Gueguen, King, Keryvin, Sangleboeuf, Rouxel, Bureau, and Lucas, 2013). The fibers were $300 \mu \mathrm{m}$ in diameter, $130 \mathrm{~mm}$ in length. The glass transition temperature of the $\mathrm{GeSe}_{9}$ glass, measured by DSC at $10^{\circ} \mathrm{C} / \mathrm{min}$, is $92^{\circ} \mathrm{C}$ (Gueguen et al., 2013). The shear relaxation and recovery tests allow for the measurements of all the distortion components during relaxation. This test is described in details in (Gueguen et al., 2013) and in references therein. The shear distortion $\gamma_{0}$ is imposed to the fiber by imposing a rotation to one of its ends (the other is fixed) with an angle $\alpha_{0}$ (here $\alpha_{0}=200 \pm 2^{\circ}$ ). The rotation angle is measured thanks to a thin needle fixed on the fiber, with a uncertainty of $\pm 2^{\circ}$. Each experimental point corresponds to a given fiber, the fibers being all tested under exactly the same conditions. The angle is imposed during a given period, up to a time " $t$ " (only $t$ varies from a fiber to another). At the time $t$ the fiber is released, the elastic distortion instantaneously recovers, and the corresponding recovered angle $\left(\alpha_{e}\right)$ is measured. Then, the delayed elastic distortion recovers over time and the corresponding additional recovered angle, evolving over time, is measured until it reaches an equilibrium value $\left(\alpha_{d}\right)$. The remaining angle $\left(\alpha_{\eta}\right)$ is due to the inelastic distortion $\left(\alpha_{0}=\alpha_{e}+\alpha_{d}+\alpha_{\eta}\right)$. It takes usually few seconds to measure an angle with the naked eye. The relationship between the distortion and the corresponding angle is (classical beam theory):

$$
\gamma_{0}=r \alpha_{0} / L \& \gamma_{c}=r \alpha_{c} / L
$$

where $c=e, d$ or $\eta$ again, $L$ the fiber length and $r$ the distance from the neutral axis of the fiber. All the distortion components are normalized by $\gamma_{0}$, so that $r$ and $L$ have no impact on the results and their uncertainty. The error bars on normalized distortions are estimated taking into account the "worst" situation: $\alpha_{c}$ is measured with an error of $+2^{\circ}$ and $\alpha_{0}$ with an error of $-2^{\circ}$ (upper limit of the error bar), and conversely (lower limit). The relaxation function corresponds to the normalized shear elastic distortion (Gueguen et al., 2013). These relaxation recovery tests have been performed at room temperature $\left(20^{\circ} \mathrm{C}\right)$. 
Glasses are, by definition, non-equilibrium materials. The ${ }_{348}$ equations tested here are only valid at equilibrium or, more precisely, if the material does not undergo aging during the 349 mechanical tests. As an example, the Ferry's relation (Eq.(15)) ${ }^{350}$ makes sense only if no aging occurs: the viscosity is not 351 time-dependent in this equation. But the viscosity, being very sensitive to the aging of the material, is actually time-dependent ${ }_{352}$ in glasses, below their glass transition temperature, until they reach their equilibrium, thanks to structural relaxation. In order to be sure that the glass is under equilibrium, we have used a specific property of some chalcogenide glasses: their photosensitivity. The glass fibers have been irradiated during two months by two light bulbs (Philips MASTER TL-D $36 \mathrm{~W} / 840$ ) that are separated from fibers by almost $1.20 \mathrm{~m}$, and the relaxation-recovery tests have been also performed under permanent irradiation. Because of a specific photoinduced process, called "photorelaxation" (Gueguen et al., 2013), the glass relaxes faster and tends to equilibrate in a photoinduced equilibrium which is not its natural configurational equilibrium (Gueguen et al., 2013). Thus, after two months, its viscosity becomes constant (Gueguen et al., 2013). It can be illustrated by the fact that the shear relaxation function remains unchanged if the test is done after two months under irradiation, and after four months under irradiation (see (Gueguen et al., 2013) and references therein). Without irradiation, at room temperature, the glass would not reach equilibrium before, at least, a decade (Gueguen et al., 2013).

These experimental conditions are chosen for the following reasons. Firstly, below the glass transition, the characteristic ${ }^{353}$ time for stress relaxation is large (here, it corresponds to ${ }^{354}$ weeks) as compared to the time required (a few seconds, so at least 5 orders of magnitude lower) for loading (to apply ${ }^{356}$ the constant strain) and unloading (to measure the elastic ${ }^{357}$ part of strain), so that we can consider that the loading and ${ }^{355}$ unloading periods do not impact on the measurements. This ${ }^{355}$ is in sharp contrast to the situation above the glass transition. ${ }^{360}$ Moreover, the full experimental setup is not instrumented ${ }^{36}$ (there are no displacement sensors or load cells) since there ${ }^{362}$ are no fast dynamics to measure; therefore it has the advantage ${ }^{366}$ of preventing any drift of sensors that could can impact on ${ }^{364}$ the measurements. Finally, at low temperatures the delayed ${ }^{365}$ elasticity is to be more exacerbated (i.e.: the stretch exponent is usually lower).

We have also performed various mechanical tests on these 370 glass fibers to assess the linearity of the viscoelastic behavior ${ }_{371}$ in the stress range investigated here (see (Gueguen et al., 2013) 372 $_{2}$ and references therein). No non-linear viscoelastic behavior is ${ }_{373}$ detectable below, at least, $55 \mathrm{MPa}$. Because the shear relax-374 ation test is a torsional test, the imposed distortion linearly in-375 creases with $r$, the distance from the neutral axis of the fiber. 376 The maximum imposed distortion, at the surface of the fiber, 377 was $\gamma_{0}=4 \pm 0.0510^{-3}$. The shear elastic modulus being ${ }_{378}$ $\mu=4.6 \mathrm{GPa}$ (see (Gueguen et al., 2013) and references therein),, 379 it corresponds to an initial stress of $\sigma(0)=18.4 \mathrm{MPa}$.

\section{Results}

The relaxation function of the $\mathrm{GeSe}_{9}$ fibers is plotted in Figure 2. The relaxation function $\varphi$ is plotted as $\ln [-\ln [\varphi(t)]] v s$. $\ln (t)$, since:

$$
\ln \left[-\ln \left[\exp \left(-\left(\frac{t}{\tau_{0}}\right)^{\beta}\right)\right]\right]=\beta \ln (t)-\beta \ln \left(\tau_{0}\right)
$$

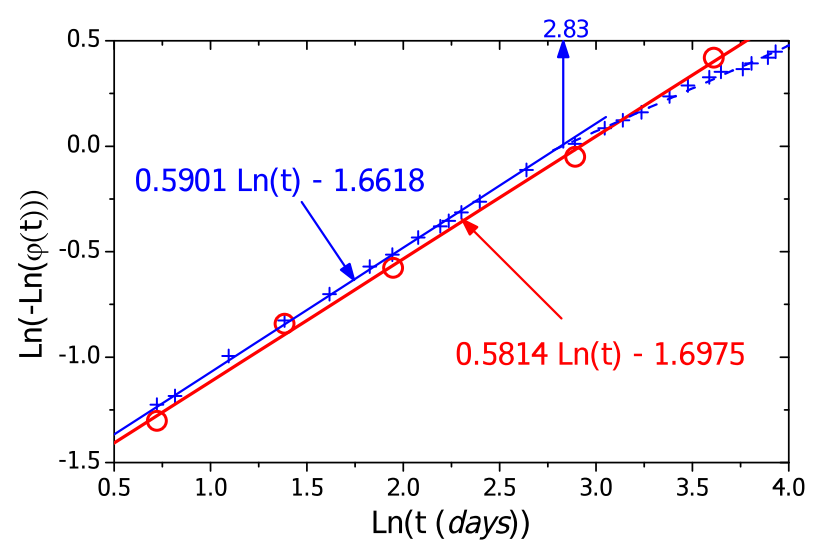

Figure 2: Experimental relaxation function obtained here (open red circle), plotted as $\ln (-\ln (\varphi(t)))$ vs. $\ln (t)(t$ is time), compared with a previous measurement (blue cross) (see (Gueguen et al., 2013) and references therein).

We obtain here, by least square fitting: $\beta=0.581$ and $\tau_{0}=18.5$ days $\left(\tau_{a}=29.1\right.$ days, according to Eq.(2)), for the five points of Figure 2. The relaxation function obtained here is compared with another one, previously obtained in the same conditions (see (Gueguen et al., 2013) and references therein). It illustrates the reproducibility of this measurement. The stretch exponent previously found was $\beta=0.59$, in very good agreement with that found here. The average relaxation time previously found was somewhat lower $\left(\tau_{a}=25.9\right.$ days, Eq.(2), with $\tau_{0}=16.8$ days), but Figure 2 illustrates that this small discrepancy corresponds to the experimental uncertainty. The shear relaxation function previously measured was bimodal (see (Gueguen et al., 2013) and references therein): for $t<17$ days, we have a first SEF, corresponding to the data plotted on Figure 2, but at $t>17$ days, the relaxation function deviates with a slower stretch exponent $(\beta=0.35)$. We can distinguish this deviation in Figure $2(\ln (17) \sim 2.83)$. The new measurements performed here do not confirm the bimodality. We do not know exactly why, and we will leave here this issue as unsolved.

The evolution of the inelastic and delayed elastic distortion, obtained from recovery tests are plotted in Figure 3. As expected from Eq.(14), the inelastic distortion continuously increases with time (the stress and the viscosity being strictly positive, $\dot{\gamma}_{\eta}$ is strictly positive). The delayed elastic distortion, instead, first increases, reaches a maximum, at $t \sim 13$ days, and then slowly decreases. It must be underlined here that the values of the inelastic and delayed elastic distortion at a time " $t$ " are obtained by starting a recovery test at this time $t$ and by 


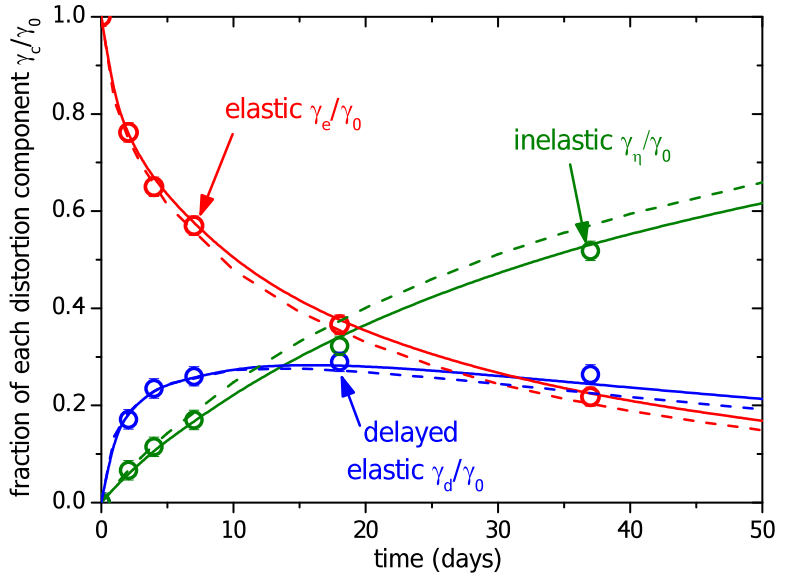

Figure 3: Evolution of each distortion contribution. The red, blue and green open circles correspond to $\gamma_{c}=\gamma_{e}, \gamma_{d}$ and $\gamma_{\eta}$, respectively. The size of the circles corresponds to the error bar. The red, green and blue lines correspond to Eq.(22), (25) and (26), respectively, with $\beta=0.58$ and $\tau_{0}=18.5$ days for the full lines, and $\beta=0.59$ and $\tau_{0}=16.8$ for the dashed lines.

waiting for the delayed elastic to fully recover (see details in (Gueguen et al., 2013)). For the last data plotted in Figure 3, this recovery part took more than one year. This is the reason why we do not have data at very long times.

In Figure 3, we have also plotted the theoretical evolutions of the delayed elastic and inelastic distortions, using Eq.(25) and (26) and the values of $\beta=0.581$ and $\tau_{0}=18.5$ identified from the experiments. As we can see, the theoretical evolutions match the experimental ones, according to the measurement uncertainty. It illustrates the validity of the equations (25) and (26). We have also plotted the theoretical evolutions with the following values, identified from the previous experiments: $\beta=0.59$ and $\tau_{0}=16.8$ days. We can see that it slightly deviates from the experimental data after $\sim 10-18$ days. Indeed, this parameter set does not allow to fit $\gamma_{d}$ and $\gamma_{\eta}$ when they also does not fit the relaxation function, since the relaxation function deviates from a single SEF after 17 days.

\section{Discussion}

\subsection{Delayed elasticity vs. stretch exponent}

The equations developed here illustrate the robustness of the $\mathrm{e}_{434}$ SEF. Using only three parameters $\left(\beta, \tau_{0}\right.$ and $\left.\mu\right)$, it is possible to ${ }_{435}$ evaluate, with a very good accuracy, the three components of ${ }_{436}$ the viscoelastic deformation, namely: the elasticity, the delayed elasticity and the inelasticity. In Figure 4, we have plotted the distortion contributions vs. the fraction of stress relaxed $\Lambda$, in ${ }^{43}$ order to illustrate the theoretical evolution of delayed elastic $c_{438}$ and inelastic distortions as a function of the stretch exponent ${ }_{439}$ $\beta$. In this Figure, we can observe that $\Lambda_{M}$, the position of the ${ }_{440}$ maximum delayed elastic distortion, is shifted to larger values 441 as $\beta$ decreases. Since $\Lambda_{M} \in \mathbb{R}^{+}$when $0<\beta<1$, the delayed ${ }_{442}$ elastic distortion first increases over time, reaches a maximum, and then decreases and tends to 0 when $t \rightarrow+\infty$. The inelastic distortion, instead, increases monotonically starting from 0 to tend to $\gamma_{0}$ when $t \rightarrow+\infty$. We can notice in Figure 4 that, if $\beta$ is low, the delayed elasticity will be the major contribution to stress relaxation, until the stress relaxes down to a very low level. The maximum relative fraction of delayed elastic distortion is $\gamma_{d}\left(\Lambda_{M}\right) / \gamma_{0}$. It is plotted $v s . \beta$ in Figure 1 . We have shown (Eq.(32)) that this maximum delayed elastic distortion is not a function of $\tau_{0}$ : it is only defined by the stretch exponent $\beta$. So, we clearly highlight here that the stretch exponent in a shear relaxation modulus is just the indicator of the delayed elastic contribution to viscoelasticity, and it is self sufficient. More precisely, the time-parameter, $\tau_{0}$, only indicates the kinetics of the relaxation, and the stretch exponent, $\beta$, only indicates the amplitude of the delayed elastic contribution to this relaxation.

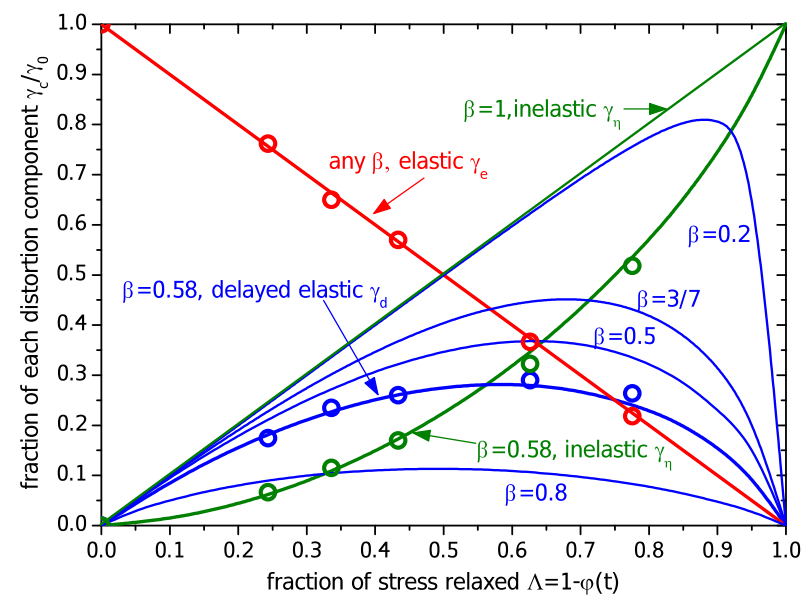

Figure 4: Theoretical evolutions (Eq.(28), (29) and (30)) of each distortion contribution $\left(\gamma_{c}=\gamma_{e}, \gamma_{d}\right.$ or $\left.\gamma_{\eta}\right)$ as a function of $\Lambda$, the fraction of stress relaxed, for various $\beta$ values. The open circles correspond to experimental data. The theoretical evolutions are also plotted for $\beta=0.59$, but they are practically indiscernible from those plotted using $\beta=0.58$.

$$
\text { there }
$$

We can note that if $\beta \rightarrow 0$, the delayed elasticity increases. Nevertheless, if $\beta=0$, there is no relaxation anymore $(\varphi(t)=$ 1 ), but the delayed elastic contribution predicted is $100 \%$. According to Eq.(6), we also observe that if $\beta \rightarrow 0$, so if $G(t) \rightarrow \mu$, that the delayed elastity is not itself able to produce full stress relaxation.

\subsection{Origin of the delayed elasticity}

Originally, the non-exponential relaxation, and so the existence of various mechanisms of stress relaxation, and corresponding energy barriers, was attributed to the inhomogeneity of glasses. There are various energy barriers first because there are various structural units (in Ge-Se glasses, 
there is $\mathrm{GeSe}_{4 / 2}$ tetrahedra, connected in different ways, Se-500 chains, probably Se-rings... (see (Yang, Gueguen, Sangleboeuf,501 Rouxel, Boussard-Pledel, Troles, Lucas, and Bureau, 2013) and $d_{502}$ references therein). But the energy barrier for a specific type of ${ }_{503}$ local relaxation event (LRE) varies from one site to another, at t504 least because of the inherent disorder of glasses (Simha, 1942).505 An atom or a structural unit can also contribute to different 506 types of LRE, with different energy barriers.

At a given temperature, under no stress, the LRE will occur 508 in random directions, producing, macroscopically, no net strain.509 Under relatively low stress, the energy barrier is slightly biased ${ }_{510}$ (not enough biased to induce non-linear viscoelasticity) favor-511 ing the relaxation events in a specific direction. These events $s_{512}$ do not necessarily correspond to global atomic displacements: ${ }_{513}$ it can correspond to a transformation or a configuration change ${ }_{514}$ as described by Argon and Kuo (1980). Indeed, during a re- ${ }_{515}$ laxation test, the strain is constant, so that there is, macroscop- ${ }_{516}$ ically, no displacement, but just a "conversion" of the initial ${ }_{517}$ elastic displacement into delayed elastic and inelastic displace- ${ }_{518}$ ments. During a relaxation test all the atoms move initially only ${ }_{519}$ "elastically" and are able to go back to their initial position. $\mathrm{As}_{520}$ soon as even a single atom (or more generally speaking a "relaxing unit", a "cooperatively rearranging subsystem" or a "cooperatively rearranging region" (Dyre, 1998)) has overcome its energy barrier to reach a new stable position/configuration the ${ }_{524}$ delayed elasticity can emerge. If the stress is released, all the ${ }_{525}$ others atoms will try to go back to their initial position, while ${ }_{526}$ this atom will not be able anymore, without overcoming again ${ }_{527}$ an energy barrier and will disturb the return of the other atoms. ${ }_{528}$ Macroscopically, the material is not able anymore to recover $_{529}$ instantaneously its initial shape. This atom is stressed by all ${ }_{530}$ other (and by reaction, stressed itself the other atoms), so that ${ }_{531}$ a driving force exists to induce its forward motion to its initial ${ }_{532}$ configuration. But since there is an energy barrier to overcome, ${ }_{533}$ this motion is delayed: this is the delayed elasticity.

Consequently, each initial LRE only produce delayed elasticity, it will be turn into inelasticity (it will be irreversible) only when its initial configuration will be not reachable anymore: when the surrounding network will have produced their own LRE to erase the memory of this initial configuration. This idea has been developed by Orowan (1952) and nicely discussed lat-536 ter by Goldstein (1969). We can go deeper into this idea by ${ }_{537}$ using a concept discussed by Argon (1968). Considering one ${ }_{538}$ specific subsystem and its corresponding LRE, with its spe-5з9 cific energy barrier (i.e.: its specific relaxation time), we un-540 derstand that if the subsystem wants to move or to change its 541 configuration, it has no reason to have exactly the free space it ${ }_{542}$ needs to do so: it will "shove" the surrounding network (Tra-543 chenko, 2007), at it is nicely depicted by the shoving-model ${ }_{544}$ of Dyre (1998), to reach a new state. The difference between ${ }_{545}$ the new and the initial states will let the surrounding network ${ }_{546}$ partially "shoved", if it can not synchronously re-arrange. $\operatorname{In}_{547}$ other words, the subsystem will occupy a new site by elasti-548 cally distorting it and stressing it or, at least will modify its549 configuration by stressing the surrounding network (let us name ${ }_{550}$ it the "shoving-stress", $\mathrm{ShS}$ ). The delayed elasticity is driven ${ }_{551}$ by the modification of the stress of the surrounding network $k_{552}$ of the subsystem (Argon, 1968). The shoving-stress (ShS) induced in the network surrounding the moving subsystems is macroscopically self-equilibrated (just like for an Eshelby's inclusion, the LRE corresponding to an eigenstrain): it does not increase the macroscopic external stress. When the strain recovery starts, this macroscopic stress becomes null, but at the microscopic scale, the ShS will slowly relax, by allowing backward motions: this is the delayed elasticity recovery. The concepts of Orowan/Goldstein or Argon have the same basis: all LRE contribute initially only to delayed elasticity, they contribute to inelasticity only because they tend to make the position/configuration changes of the previous LRE irreversible.

During a relaxation test, the macroscopic stress applied relaxes, the elastic energy initially stored being dissipated during LRE (Maxwell, 1868). Each event induces a local ShS, increasing the amount of delayed elasticity. Nevertheless, since this $\mathrm{ShS}$ can also relax, there is a competition between a creation and a relaxation of the ShS. Accordingly, the delayed elasticity, during a relaxation test, increases and reaches a maximum before decreasing. This is illustrated here on Figure 3. During a creep test, the delayed elasticity created saturates when the rate of creation of ShS equilibrates with its rate of relaxation (Argon, 1968). During a relaxation test, each LRE decays the stress, that is not fully redistributed to the surrounding network. During a creep test, a subsystem bears less stress after a LRE, but all the stress it does not bear anymore is fully redistributed on the surrounding network Just imagine a truss made of various beams, and break a beam of this truss (by analogy to a LRE): if the truss is bearing a constant load, all the other beams will support more load to exactly compensate the load the broken beam was bearing, and will deform more (this is the creep test). If a constant distortion is imposed to the truss, once a beam is broken, the truss is less rigid, the load needed to impose the same distortion will globally decrease (this is the relaxation test).

\subsection{Generalization to any kind of relaxation functions}

There are two standard ways to model a linear viscoelastic body by using cells made of springs and dashpots. The first one is to use "parallel" models, such as the Maxwell-Wiechert model, where there is no individual cell that induces delayed elasticity. The second one is to use "serial" models, such as the Burgers's model, where the delayed elasticity is usually due to Kelvin-Voigt cells. For this latter solution, we have cells to model the delayed elasticity, but without clear microscopical explanation for it: we could assume that some LRE (associated with Kelvin-Voigt cells) induce exclusively delayed elasticity and some others induce exclusively viscous flow, without interacting with each other. But serial models can just be considered as other kind of representations of parallel models. For the parallel models, we can assume that a relaxation function can be decomposed as a weighted sum of exponential processes, each of these processes having its own relaxation time. Let us assume a finite number $(N)$ of relaxation processes (a discontinu- 
ous relaxation spectrum) having an additive contribution:

$$
\varphi(t)=\sum_{i=1}^{N} \rho_{i} \exp \left(-\frac{t}{\tau_{i}}\right)
$$

$\rho_{i}$ is the weight of the $i^{\text {th }}$ process having a relaxation time ${ }^{594}$ $\tau_{i}$, verifying $\sum_{i=1}^{N} \rho_{i}=1$ (this is the Maxwell-Wiechert model ${ }^{595}$ (Wiechert, 1893)).

Let $n(t)$ be the numbers of LRE that have already occurred ${ }^{598}$ at $t$ and $n_{T}$ is the total number of LRE that will occur until the599 stress becomes null. The LRE producing the stress relaxation, 600 according to the definition of the relaxation function, we have: 601

$$
\frac{n(t)}{n_{T}}=1-\varphi(t)=\sum_{i=1}^{N} \rho_{i}\left(1-\exp \left(-\frac{t}{\tau_{i}}\right)\right)
$$

Among the $n$ LRE, a part of them have induced motions that ${ }^{606}$ have let some subsystems in configurations where they can pro- ${ }^{607}$ duce delayed elasticity. These LRE can be considered as "re- ${ }^{608}$ versible", since the corresponding backward events occur when ${ }^{600}$ the macroscopic stress is removed. Let $n_{r}$ be the number of such ${ }^{610}$ LRE. It is proportional to the delayed elastic strain. The other ${ }^{611}$ part of the LRE produces viscous flow, they have let subsys ${ }^{612}$ tems in stable configurations, they are "irreversible", since no ${ }^{613}$ backward motion is possible. Their number is $n_{i}$, proportional ${ }^{614}$ to the viscous strain. So, we have:

$$
n(t)=n_{r}(t)+n_{i}(t)
$$

We first derive this expression, using Eq.(23), $n_{i} / n_{T}$ being ${ }^{619}$ equivalent to $\gamma_{\eta} / \gamma_{0}$, according to Eq.(36):

$$
\dot{n}_{i}(t)=n_{T} \frac{\dot{\gamma_{\eta}}}{\gamma_{0}}=n_{T} \frac{\gamma_{0} \mu \varphi(t)}{\gamma_{0} \eta}=\frac{n_{T}-n(t)}{\tau_{a}}
$$

Then we directly obtain $i_{r}(t)$. After integration, and assum-625 ing that $n_{r}(0)=0$, it leads to the fraction of "reversible LRE": 626

$$
\frac{n_{r}(t)}{n_{T}}=\sum_{i=1}^{N}\left(1-\frac{\tau_{i}}{\tau_{a}}\right) \rho_{i}\left(1-\exp \left(-\frac{t}{\tau_{i}}\right)\right)
$$

Now, if we assume a continuous relaxation spectrum:

$$
\varphi(t)=\int_{0}^{+\infty} \rho(\tau) \exp \left(-\frac{t}{\tau}\right) d \tau
$$

Here, the weight $\rho$ is per time unit $\left(d(\tau)=\rho(\tau) \tau\right.$ is the dis- ${ }^{633}$ tribution function of relaxation times), and $\rho \geq 0$. According ${ }^{634}$ to Eq.(2), we have: $\tau_{a}=\int_{0}^{+\infty} \rho(\tau) \tau d \tau$. Note that for a SEF, $\beta$ is a monotonous function of the logarithmic full width at half maximum of $d(\tau)$ (Johnston, 2006), the distribution function of relaxation times. And we obtain, by analogy with the MaxwellWiechert model:

$$
\frac{n_{r}(t)}{n_{T}}=\int_{0}^{+\infty}\left(1-\frac{\tau}{\tau_{a}}\right) \rho(\tau)\left(1-\exp \left(-\frac{t}{\tau}\right)\right) d \tau
$$

And the fraction of "irreversible LRE":

$$
\frac{n_{i}(t)}{n_{T}}=\int_{0}^{+\infty} \frac{\tau}{\tau_{a}} \rho(\tau)\left(1-\exp \left(-\frac{t}{\tau}\right)\right) d \tau
$$

These two latter equations explicitly show that the delayed elasticity (the reversible LRE) directly comes from the difference between the relaxation time $\tau$ (or $\tau_{i}$ if the spectrum is discontinuous) of a specific LRE and the average relaxation time $\tau_{a}$. The relative proportion of reversible events produced by a process having relaxation times between $\tau$ and $\tau+d \tau$ is: $\left(1-\frac{\tau}{\tau_{a}}\right) \rho(\tau) d \tau$. Obviously, if all the LRE have exactly the same relaxation time $\left(\tau=\tau_{a}\right)$, we obtain $n_{r}(t)=0$ and $n_{i}(t)=n(t)$. None of the LRE produces reversible LRE, all are irreversible. So whatever the relaxation function based on a given distribution $d(\tau)$ used, the delayed elasticity comes from a "dispersion" of the relaxation times. Conversely, some viscoelastic bodies can exhibit delayed elasticity without viscous flow, they are called "viscoelastic solids" (as oppose to viscoelastic liquid (Lakes, 1998)). For such bodies, we may assume that some slow processes have very large relaxation times as compare to fast ones, enough large to consider that, at the human scale, they can be set as infinite (relative Deborah number $\gg 1$ ). Thus, if at least the $j^{\text {th }}$ relaxation time $\left(\tau_{j}\right)$ in Eq.(35), is set as infinite, then $\varphi(t)=\rho_{j}$ when $t \rightarrow+\infty$. Additionally, according to Eq.(42), $n_{i} \rightarrow 0$, at any time, since $\tau_{a} \rightarrow+\infty$. In other words, bodies with infinitely dispersed relaxation times, but at least with one finite relaxation time, exhibit only delayed elasticity.

The equations (41) and (42) are in perfect agreement with the concepts proposed by Orowan (1952), Goldstein (1969) and Argon (1968). All initial and "fast" LRE, with small relaxation times $\left(\tau \leq \tau_{a}\right)$, first mainly induce delayed elasticity (they induce reversible events: $n_{r}>0$ ): their initial position/configuration/state are still reachable. Then, the "slow" LRE, with large relaxation times $\left(\tau \geq \tau_{a}\right)$, occur and tend to make, by their own configuration/position changes, the initial states of fast LRE unattainable (they annihilate the reversibility of the first LRE: $n_{r}<0$ ). It is easy to see that "slow" LRE only annihilate the reversibility of the events produced by "fast" LRE, since their respective contributions to $n_{r}$ exactly compensate each other. Indeed, the term $\left(1-\frac{\tau}{\tau_{a}}\right) \rho(\tau)$ satisfies:

$$
\int_{0}^{+\infty}\left(1-\frac{\tau}{\tau_{a}}\right) \rho(\tau) d \tau=0
$$

We can provide an illustration of these equations using a simple $\rho(\tau)$, for which we will have only analytical solutions (Johnston, 2006):

$$
\rho(\tau)=\frac{\exp \left(-\frac{\tau}{4 \tau_{0}}\right)}{2 \sqrt{\pi \tau \tau_{0}}} \rightarrow \varphi(t)=\exp \left(-\left(\frac{t}{\tau_{0}}\right)^{1 / 2}\right)
$$

With this $\rho(\tau)$, we have $\tau_{a}=2 \tau_{0}$. We set $n_{T}=1$ in order to normalize the functions. Then we obtain the contribution $\left(n_{r}^{+}\right)$ of the fast processes $\left(\tau \leq \tau_{a}\right)$ to $n_{r}$, by replacing " $+\infty$ " in the integral of Eq.(41) by $\tau_{a}$, and the contribution $\left(n_{r}^{-}\right)$of the 


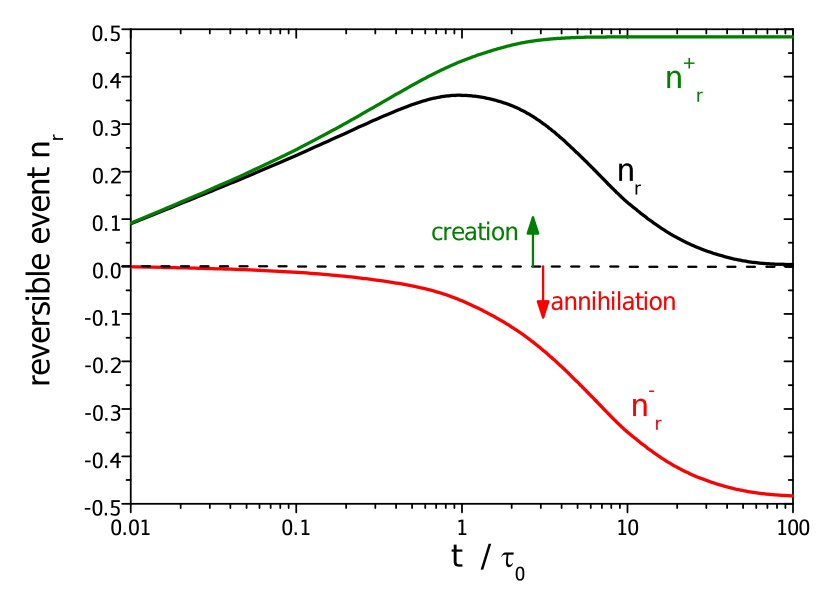

slow processes $\left(\tau \geq \tau_{a}\right)$ to $n_{r}$ by replacing " 0 " in the integral 672 of of Eq.(41) by $\tau_{a} . n_{r}^{+}$and $n_{r}^{-}$are plotted on Figure 5 with the 673 total amount of $n_{r}$. Note that $n_{r}$ is directly found using Eq.(41) $)_{674}$ but also using Eq.(26), these two equations being analytically 675 strictly equivalent. We clearly see on this Figure that the total amount of $n_{r}=n_{r}^{+}+n_{r}^{-}$(and thus the delayed elasticity) is only due to the difference between the rate of creation of $n_{r}$ by the fast processes and the rate of annihilation of these $n_{r}$ by the slow one. time (normalized by $\tau_{0}$ ) for a SEF with $\beta=1 / 2$ of the amount of reversible ${ }_{697}$ LRE $\left(n_{r}^{+}\right)$produced by all the processes having relaxation times lower than $\tau_{a}{ }^{698}$ (green line) and of the amount of reversible LRE $\left(n_{r}^{-}\right)$annihilated by all the 699 processes having relaxation times larger than $\tau_{a}$ (red line).

\subsection{Models without initial distribution of relaxation times}

Trachenko (2007) is, to our knowledge, the only one propos- ${ }^{704}$ ing a model corresponding to a SEF, where no pre-set distri- ${ }^{705}$ bution of relaxation time $(\rho(\tau))$ exists. In this model, since ${ }^{700}$ a relaxing unit "support less stress after relaxation, later $\mathrm{LRE}^{707}$ should support more stress in order to counterbalance" (Tra- ${ }^{708}$ chenko, 2007), thus increasing their energy barrier (actually, ${ }_{710}$ since the macroscopic stress relaxes, the later LRE will rather ${ }^{710}$ support less stress, they should instead support a new stress field, self equilibrated). Consequently, the relaxation time of the LRE increases with the number of LRE that have already $y_{712}$ occurred, leading to a stretched exponential decay of the macroscopic stress. One consequence of the model is that the stretch ${ }^{713}$ exponent is inversely proportional to the stress decay $\left(\Delta p_{0}\right)_{715}^{714}$ due to a LRE (Trachenko, 2007) and thus to the initial ap-716 plied stress $\left(\sigma(t=0)=\sigma_{0}\right)$. Indeed, the model assumes ${ }^{717}$ that all LRE induce the same $\Delta p_{0}$ (Trachenko, 2007), so that: ${ }^{718}$ $\sigma(t)=\left(n_{T}-n(t)\right) \Delta p_{0}$ and $\Delta p_{0}=\sigma_{0} / n_{T}$, the final number of ${ }_{720}^{719}$ LRE $\left(n_{T}\right)$ at the end of the relaxation being stress independent $t_{721}$ (Trachenko, 2007). Thus, this model can clearly not be applied ${ }^{722}$ as a relaxation function for linear viscoelastic materials, such $^{723}$ as the glass investigated here, since the relaxation function is $_{725}^{724}$ stress dependent. Since we do not know other models of stress 726 relaxation in the framework of linear viscoelasticity, without pre-set distribution of relaxation times, we can assume that, in that framework, the delayed elasticity can just be seen as a consequence of the broadness of the distribution.

\section{Conclusion}

According the idea of Wiechert, the delayed elasticity takes its origins in the non-exponentiality of the stress relaxation, and thus, is due to a dispersion of relaxation times of all the processes involved during relaxation. In spite of the fact that the SEF is not physically sound and can not be used alone (without an other model at $t \rightarrow 0^{+}$) as a phenomenological model (Duffrène et al., 1997), the experimental investigation done here highlights that this function can be perfectly suitable to describe the detailed viscoelastic deformation of a linear viscoelastic material. The equations developed here highlight the role of delayed elasticity during relaxation and shows that the maximum delayed elasticity reached during relaxation is only correlated to the stretch exponent $(\beta)$. This maximum continuously increases as $\beta$ decreases. Actually, the delayed elasticity is the result of the broadness (connected to $\beta$ for a SEF) of the distribution function of relaxation times, whatever this function. For a distribution function $d(\tau)$, the relative delayed elasticity induced $(>0)$ or annihilated $(<0)$ by the process having a relaxation time $\tau$ is: $\left(1-\tau / \tau_{a}\right) d(\tau)$, where $\tau_{a}$ is the average relaxation time of the whole system. At short time, the subsystems having low relaxation times induce large amount of delayed elasticity by moving in a network of slow subsystems that can only accommodate the motions elastically. At long time, these slow subsystems, by moving, accommodate inelastically the motions of the fast subsystems and annihilate the delayed elasticity by turning it into inelasticity.

Additionally, since, to our knowledge, no inorganic glasses exhibits or even approach a Maxwell-Debye stress relaxation $(\beta \rightarrow 1)$ until they are far above their glass transition temperature $\left(T_{g}\right)$, all of them exhibit delayed elasticity below and above $T_{g}$. Accordingly, viscosity measurements in this range must be isothermal, to reach the stationary creep stage, or they will be just absolutely wrong. Viscosity measurements made during a continuous heating, just give almost something close to the initial delayed elastic strain rate.

\section{References}

Argon, A., Kuo, H., 1980. Free energy spectra for inelastic deformation of five metallic glass alloys. J. Non-Cryst. Solids 37 (2), $241-266$.

Argon, A. S., 1968. Delayed elasticity in inorganic glasses. J. Appl. Phys. 39 (9), 4080-4086.

ASTM C1350-96, 1996. Standard test method for measurement of viscosity of glass between softening point and annealing range (approximately $10^{8} \mathrm{~Pa}$ - s to approximately $10^{13} \mathrm{~Pa}-\mathrm{s}$ ) by beam bending (metric). It says : "the method also may be used in experimental programs that focus on nonequilibrium conditions", whereas only the measurement of the delayed elasticity allow such investigation.

Bendler, J. T., 1984. Levy (stable) probability densities and mechanical relaxation in solid polymers. Journal of Statistical Physics 36 (5-6), 625-637.

Boltzmann, L., 1876. On the theory of the elastic aftereffect. Pogg. Ann. Erg. Bd. 7, 624-645. 
Duffrène, L., Gy, R., Burlet, H., Piques, R., 1997. Viscoelastic behavior of a soda-lime-silica glass: inadequacy of the KWW function. J. Non-Cryst. Solids 215 (2-3), 208-217.

Dyre, J. C., 1998. Source of non-arrhenius average relaxation time in glassforming liquids. J. Non-Cryst. Solids 235-237, 142-149.

Ferry, J. D., 1980. Viscoelastic properties of polymers. Vol. 3. Wiley New York. Frenkel, J., 1926. Über die wärmebewegung in festen und flüssigen körpern. Zeitschrift für Physik 37 (7-8), 572-609.

Goldstein, M., 1969. Viscous liquids and the glass transition: a potential energy barrier picture. J. Chem. Phys. 51, 3728-3739.

Gueguen, Y., King, E. A., Keryvin, V., Sangleboeuf, J.-C., Rouxel, T., Bureau, B., Lucas, P., 2013. Photoinduced aging and viscosity evolution in se-rich Ge-Se glasses. J. Appl. Phys. 114, 074901.

Johnston, D. C., Nov 2006. Stretched exponential relaxation arising from a continuous sum of exponential decays. Phys. Rev. B 74, 184430.

Kohlrausch, R., 1854. Pogg. Ann. Phys. 91, 198.

Lakes, R. S., 1998. Viscoelastic solids. CRC press.

Lee, E. H., 1955. Stress analysis in viscoelastic bodies. Quarterly Applied Mathematics 13, 183-190.

Maxwell, J., 1868. On the dynamical theory of gases. Phil. Mag. 35, 134.

Orowan, E., 1952. "Creep in metallic and non-metallic materials". Proceedings of the First National Congress of Applied Mechanics (American Society of Mechanical Engineers, New York), 453.

Phillips, J. C., 1996. Stretched exponential relaxation in molecular and electronic glasses. Reports on Progress in Physics 59 (9), 1133.

Simha, R., 1942. On relaxation effects in amorphous media. J. Appl. Phys. 13 (3), 201-207.

Trachenko, K., Jun 2007. Slow dynamics and stress relaxation in a liquid as an elastic medium. Phys. Rev. B 75, 212201.

Weber, W., 1835. Über die elastizität der seidenfäden. Ann. Phys. 34, 247-257. Wiechert, E., 1893. Gesetze der elastischen nachwirkung für constante temperatur. Annalen der Physik 286 (11 \& 10), 335-348 \& 546-570.

Yang, G., Gueguen, Y., Sangleboeuf, J., Rouxel, T., Boussard-Pledel, C., Troles, J., Lucas, P., Bureau, B., 2013. Physical properties of the $\mathrm{Ge}_{x} \mathrm{Se}_{1-x}$ glasses in the $0<x<0.42$ range in correlation with their structure. J. NonCryst. Solids 377, 54-59.

Zener, C., 1948. Elasticity and Anelasticity of Metals. University of Chicago Press, Chicago. 